\title{
Role of C11-FDG dual-tracer PET-CT scan in metastatic screening of hepatocellular carcinoma-a cost-effectiveness analysis
}

\author{
Kevin K. W. Chu, Albert C. Y. Chan, Ka Wing Ma, Wong Hoi She, Wing Chiu Dai, Kenneth S. H. Chok, \\ Tan To Cheung, Chung Mau Lo \\ Department of Surgery, The University of Hong Kong, Hong Kong, China \\ Contributions: (I) Conception and design: ACY Chan; (II) Administrative support: ACY Chan; (III) Provision of study materials or patients: All \\ authors; (IV) Collection and assembly of data: KKW Chu, ACY Chan; (V) Data analysis and interpretation: KKW Chu, ACY Chan; (VI) Manuscript \\ writing: All authors; (VII) Final approval of manuscript: All authors. \\ Correspondence to: Albert C. Y. Chan, MS, FRCS. Department of Surgery, The University of Hong Kong, 102 Pok Fu Lam Road, Hong Kong, \\ China. Email: acchan@hku.hk.
}

Background: We aimed to identify predictive factors for positron emission tomography (PET)-detected hepatocellular carcinoma (HCC) metastasis and a cost-effective approach to preoperative PET-computed tomography (CT) for detecting metastasis.

Methods: Clinicopathological and survival data of HCC patients having PET-CT with 18F-fludeoxyglucose (FDG) and 11C-acetate (ACT) following contrast-enhanced CT/magnetic resonance imaging (MRI) for preoperative tumor staging were reviewed. Binary logistic regression was performed to identify predictive factors for PET-detected metastasis. A cost-benefit analysis model was built for the incurred costs and the impact of PET-CT findings on treatment strategy was studied.

Results: Totally 152 patients were analyzed. Dual-tracer PET-CT detected metastasis in 17 patients (11\%). By multivariate analysis, alpha-fetoprotein (AFP) $\geq 400 \mathrm{ng} / \mathrm{mL}$ [relative risk (RR): 4.30, 95\% confidence interval (CI): 1.41-13.15, P=0.011] and bilobar disease (RR: 3.94, 95\% CI: $1.24-12.52, \mathrm{P}=0.014$ ) were independent predictive factors for PET-detected metastasis. PET-CT findings altered the treatment strategy for 12 patients (7.9\%); three partial hepatectomies, eight episodes of transarterial chemoembolization (TACE) and one episode of ablation were avoided, with an estimated cost-saving of US \$91,000, \$150,000 and \$10,600 respectively. Had the PET-CT been performed only for patients with AFP $\geq 400 \mathrm{ng} / \mathrm{mL}$ or bilobar disease $(\mathrm{n}=74)$, metastasis would have been confirmed in 14 patients $(18.9 \%)$, and the cost-saving per patient was estimated at US $\$ 1,070$.

Conclusions: Dual-tracer PET-CT is cost-effective and useful for preoperative HCC staging in patients with AFP $\geq 400 \mathrm{ng} / \mathrm{mL}$ or bilobar disease. Its routine use in preoperative workup for all HCC patients is not recommended. Unilobar disease with AFP $<400 \mathrm{ng} / \mathrm{mL}$ can achieve good negative predictive value for PETdetected metastasis. Screening patients with either factor can avoid unnecessary procedures and is thus costeffective for preoperative HCC workup.

Keywords: Cost-effectiveness study; dual-tracer positron emission tomography-computed tomography (dualtracer PET-CT); hepatocellular carcinoma (HCC); metastatic screening; preoperative investigation

Submitted Jun 04, 2019. Accepted for publication Aug 29, 2019.

doi: 10.21037/hbsn.2019.11.09

View this article at: http://dx.doi.org/10.21037/hbsn.2019.11.09 


\section{Introduction}

Hepatocellular carcinoma (HCC) is the sixth most common cancer and the third most common cause of cancer-related deaths worldwide (1). HCC diagnosis is difficult in some cases with small or atypical enhancing patterns on computed tomography (CT) and magnetic resonance imaging (MRI). Dual-tracer positron emission tomography (PET)-CT with 18F-fludeoxyglucose (FDG) and 11C-acetate (ACT) has been reported to have high sensitivity and specificity to detect primary HCC and its metastases (2). The usefulness of dual-tracer PET-CT in primary tumor and metastasis has been shown in previous studies (2-4). The pattern of tracer uptake by tumors is correlated with tumor cellular differentiation (3). Hence, dual-tracer PET-CT has been increasingly employed as the choice of imaging modality for preoperative tumor staging in all patients with potentially resectable HCC. However, there is a scarcity of data on the efficacy and cost-effectiveness of such an approach. The aim of this study is to investigate if routine dual-tracer PET scan for preoperative staging of HCC is oncologically and financially justifiable. We present the following article in accordance with the CHEERS reporting checklist (available at https://hbsn. amegroups.com/article/view/10.21037/hbsn.2019.11.09/rc).

\section{Methods}

The study was conducted in accordance with the Declaration of Helsinki (as revised in 2013). This study has been approved by the Institutional Review Board of the University of Hong Kong/Hospital Authority Hong Kong West Cluster (IRB Reference Number: UW 19315). The study was a review and analysis of treatment costs; treatments received by the patients were not affected by this retrospective study and no individual patient can be identified by the data reported in the study. As such, patients' consent to the study was not required.

Prospectively collected data of patients who were referred for HCC workup in the period from December 2012 to September 2015 at our center were reviewed. Routine pre-treatment staging for HCC entailed contrastenhanced CT or MRI followed by dual-tracer PET scan. Patients with interval period between two imaging less than 12 weeks were included The purpose of using a dual radioisotope with $11 \mathrm{C}-\mathrm{ACT}$ and $18 \mathrm{~F}-\mathrm{FDG}$ has been elucidated by previous study $(3,5)$. Briefly, 11C-ACT was injected intravenously at $7.4 \mathrm{MBq} / \mathrm{kg}$ and whole-body imaging was performed at 20 minutes after injection.
Non-contrast CT was performed followed by PET with a 2 minutes emission acquisition time. Fifteen minutes after the 11C-ACT imaging (approximately 45 minutes after initial 11C-ACT injection), 18F-FDG radiotracer will be injected at $6.3 \mathrm{MBq} / \mathrm{kg}$ intravenously. Sixty minutes after the injection of $18 \mathrm{~F}-\mathrm{FDG}$ radiotracer, imaging with the same position and settings will be performed with PET and CT using the same reconstruction parameters and specification as stated earlier. HCC treatment featured surgical resection, radiofrequency ablation or transarterial chemoembolization (TACE). Patients with recurrent HCC or other malignant liver pathology were excluded.

Nonparametric analysis was conducted. The data were expressed as medians with ranges. Comparisons between groups were performed with the Mann-Whitney $\mathrm{U}$ test. Discrete variables were compared by Pearson's chi-squared test or Fisher's exact test where appropriate. Sensitivity, specificity, positive predictive value and negative predictive value were calculated. Univariable and multivariable predictors of metastasis were calculated. $\mathrm{P}$ values $<0.05$ were considered to be statistically significant. The computer software SPSS 22 for Windows was used for all statistical analyses.

A cost-benefit analysis model was built with the costs and the consequence of change in treatment strategy. All direct cost of standard staging with CT and PET-CT and estimated cost of liver surgery, hospitalization and other local treatment for HCC were taken into consideration for cost analysis. All monetary values were expressed in US dollars.

\section{Results}

During the study period, 209 patients with PET-CT performed for HCC was referred to our center. Among them, 13 patients had treatment before the imaging and 24 patients had incomplete imaging. After excluding these patients, 152 patients entered our analysis (Figure 1).

Dual-tracer PET-CT scan was performed in 73 patients for pre-treatment tumor staging (group 1) and in 79 patients with atypical CT/MRI features of HCC for diagnostic purpose as well as screening for metastasis (group 2). Since all patients in group 2 had HCC except one patient whose disease was histologically confirmed to be cholangiocarcinoma after resection, the two groups of patients were combined for analysis. Seventeen patients were found to have distant metastasis. Among them, 6 patients had lung metastasis, 7 had bone metastasis, and 4 


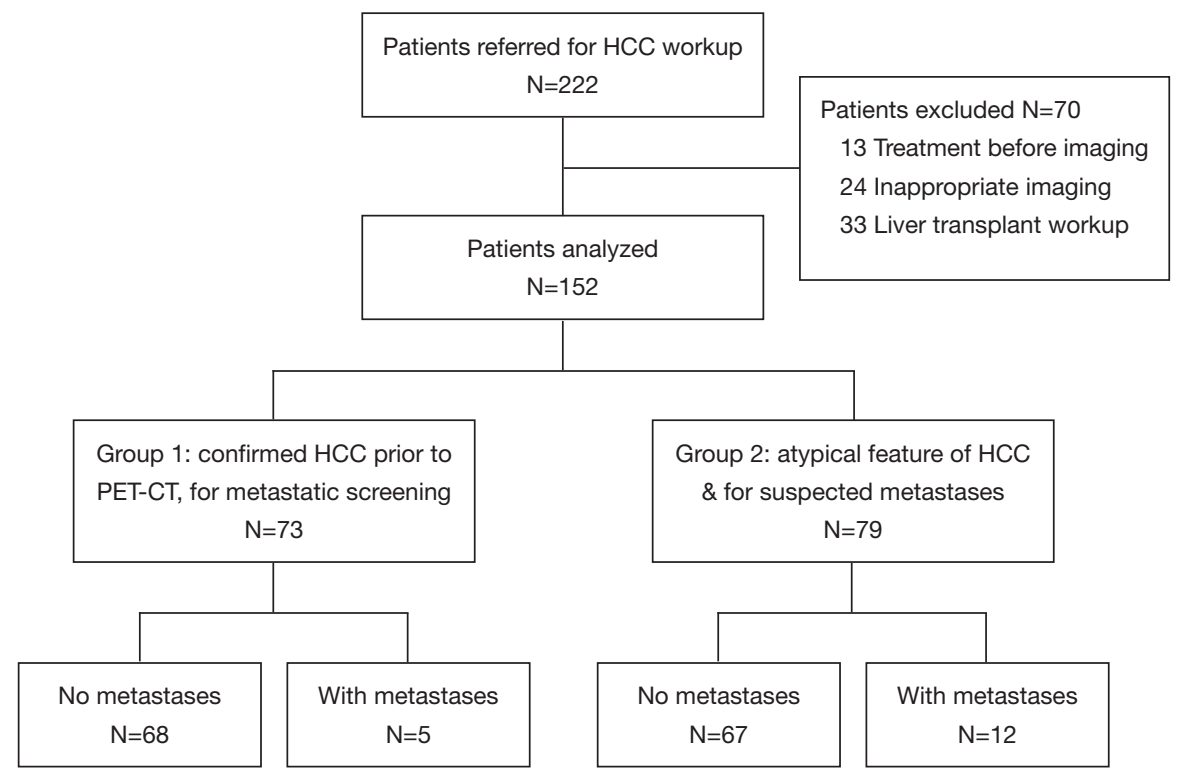

Figure 1 Patient inclusion and grouping. HCC, hepatocellular carcinoma; PET, positron emission tomography; CT, computed tomography.

had distant lymph node metastasis. Patients with metastatic disease had higher Child-Pugh grading, larger tumor size, multifocal or bilobar HCC, and higher alpha-fetoprotein (AFP) (Table 1). None of the patients had their tumors upstaged or down-staged due to dual-tracer PET-CT findings.

By univariate analysis, Child-Pugh class A was found to be a negative predictive factor for metastasis [relative risk (RR): $0.226,95 \%$ confidence interval (CI): 0.075-0.675, $\mathrm{P}=0.008$ ]. Multifocality (RR: 4.45 , 95\% CI: $1.38-14.35$, $\mathrm{P}=0.013$ ), bilobar HCC (RR: 5.50, 95\% CI: 1.82-16.63, $\mathrm{P}=0.003$ ) and $\mathrm{AFP} \geq 400 \mathrm{ng} / \mathrm{mL}$ (RR: $5.61,95 \% \mathrm{CI}: 1.93-$ $16.35, \mathrm{P}=0.002$ ) were shown to be predictive factors for metastasis. Further multivariate analysis showed that only AFP $\geq 400 \mathrm{ng} / \mathrm{mL}$ (RR: $4.30,95 \%$ CI: $1.41-13.15, \mathrm{P}=0.011$ ) and bilobar HCC (RR: 3.94, 95\% CI: 1.24-12.52, $\mathrm{P}=0.014$ ) were independent predictive factor for metastasis (Table 2).

Taking serum AFP and bilobar disease into consideration, if both factors were present, patients would have developed distant metastasis. On the other hand, when both clinicopathological factors were absent, only 3 patients (96\% negative predictive value) had distant metastasis (Table 3).

A basic cost-effective analysis was performed with all direct costs of PET/CT, hepatectomy, hospital stay, and other treatment. The mean cost of hepatectomy, ablation and TACE were $\$ 25,600, \$ 3,840$ and $\$ 6,400$ respectively. The mean cost of in-patient hospital stay was $\$ 600 /$ day and the median stay for partial hepatectomy, TACE and ablation were $8,4,7$ days respectively. The cost of dualtracer PET/CT was $\$ 1,920$ per patient. To calculate the cost-effectiveness of PET/CT, each patient was investigated independently and management decision was made before PET/CT. Twelve patients (among the 17 patients with metastasis) had their management altered after PET/CT. With the use of PET/CT, unnecessary treatments could be avoided: hepatectomy for 3 patients costing $\$ 91,000$, TACE for 8 patients costing $\$ 150,000$, and ablation for one patient costing $\$ 10,600$. The total cost of these interventions was \$251,000, while the total cost of PET/CT for 152 patients was $\$ 292,000$ (Table 4). Applying the multivariant analysis with the factor AFP $\geq 400 \mathrm{ng} / \mathrm{mL}$ or bilobar involvement as a criterion for performing PET/CT, we could reduce the use of PET/CT to 74 patients. Among these 74 patients, 10 patients had their management altered, and the cost of procedures avoided ( 2 hepatectomies, 7 TACE, 1 ablation) amounted to $\$ 215,000$. The total cost of PET/CT for 74 patients amounted to $\$ 136,000$.

With the above analysis, performing PET/CT for patients with risk factors could save patients from unnecessary procedures and also an amount of $\$ 79,000$ for the group of 74 patients.

\section{Discussion}

Dual-tracer PET-CT with 11C-ACT and 18F-FDG has been found to be useful in the evaluation of primary and 
Table 1 Patient demographics

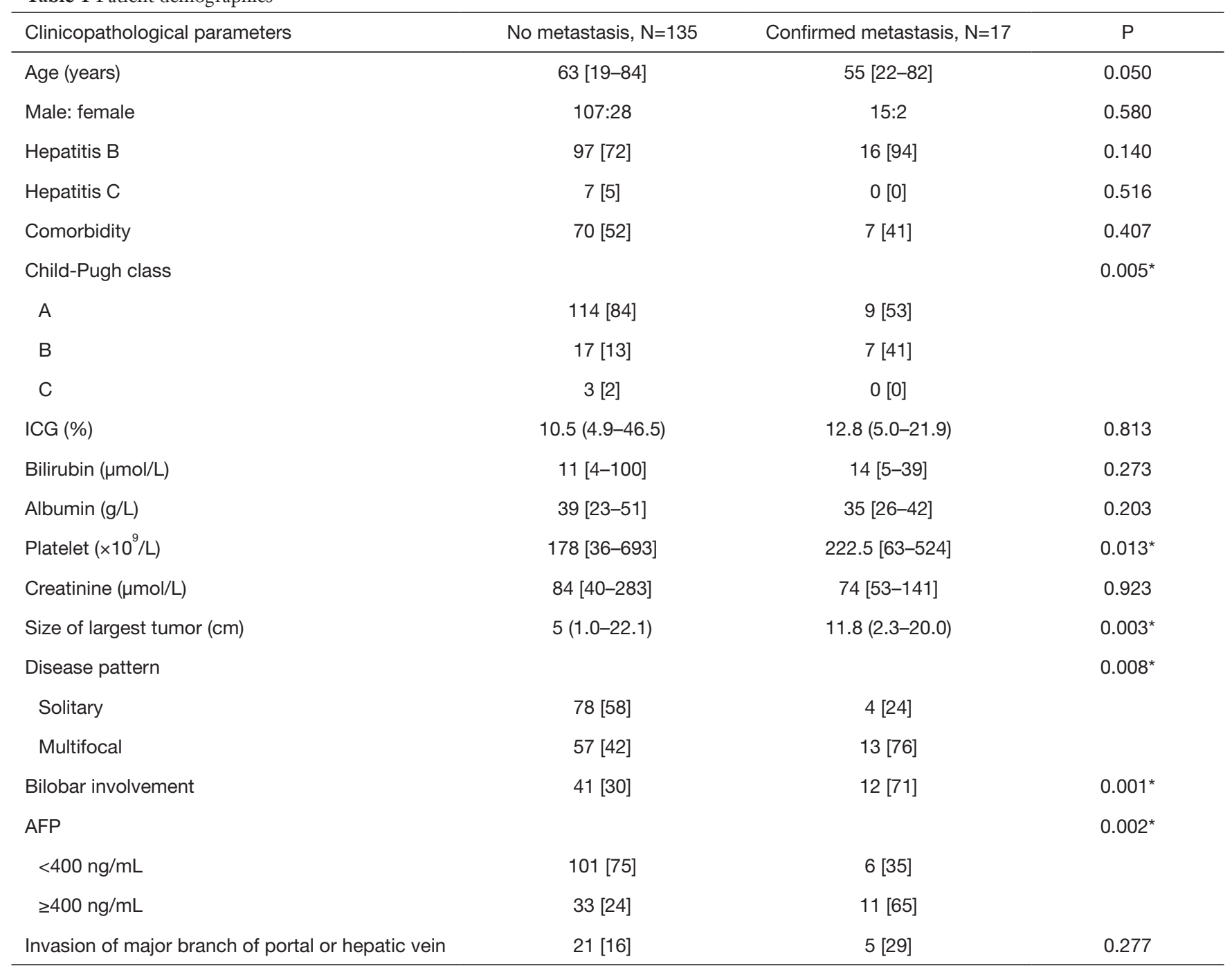

Data in median [range] or number of patients [percent] or otherwise stated. *, statistically significant. ICG, indocyanine green retention rate at 15 minutes after injection; AFP, alpha-fetoprotein.

metastatic HCC (2-4). Studies also showed that dual-tracer PET-CT had high sensitivity and specificity to detect extrahepatic metastases (2). However, patient selection criteria for dual-tracer PET need to be defined. Dual-tracer PET is an expensive investigation and it would be illogical to use this imaging modality for all pre-treatment staging in HCC. Our current study showed that only tumors with AFP $\geq 400 \mathrm{ng} / \mathrm{mL}$ or bilobar involvement were true indications for dual-tracer PET scan, owing to the incidence of distant metastasis. Furthermore, cost-effectiveness was enhanced when only patients with aforementioned tumor features were selected for this investigation.

It has been reported that dual-tracer PET-CT was better at characterizing liver lesions due to the preferential uptake of 11C-ACT (3), a feature of well-differentiated HCC. In our current study, however, this benefit was not demonstrated. Seventy-nine patients in the study had atypical primary imaging features on initial imaging, e.g., absence of arterial enhancement or portovenous washout. Surprisingly, all but one of them turned out to have HCC. The exception was found to have cholangiocarcinoma on histopathological examination but his surgical management was performed as if he had HCC. Our center is a tertiary referral center for hepatobiliary and pancreatic surgery. The patients referred to our clinic were seen by primary care takers and were considered to have a high suspicion 
Table 2 Analyses for predictive factors for PET-detected metastasis

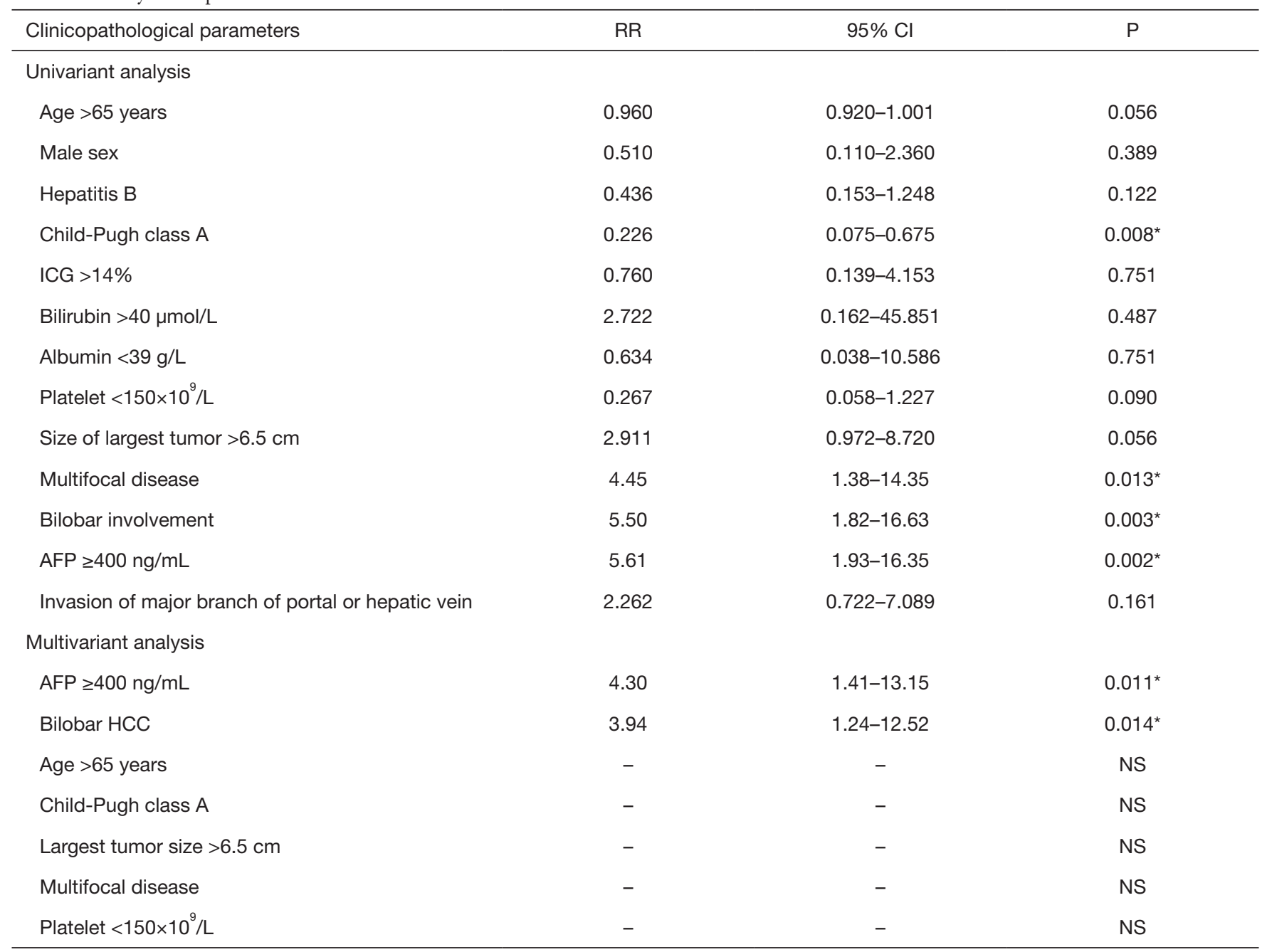

*, statistically significant. PET, positron emission tomography; RR, relative risk; Cl, confidence interval; ICG, indocyanine green retention rate at 15 minutes after injection; NS, not significant; HCC, hepatocellular carcinoma; AFP, alpha-fetoprotein.

Table 3 Accuracy of multivariable predictors in screening metastasis

\begin{tabular}{|c|c|c|c|c|}
\hline Predictors & \multicolumn{2}{|c|}{ Metastasis } & Positive predictive value & Negative predictive value \\
\hline Either + & 14 & 59 & $19 \%(14 / 73)$ & - \\
\hline Both - & 3 & 78 & - & $96 \%(78 / 81)$ \\
\hline Sensitivity & $82 \%(14 / 17)$ & - & - & - \\
\hline
\end{tabular}


Table 4 Costs of PET/CT and procedures avoided, analysis for all patients and with predictive criteria applied

\begin{tabular}{|c|c|c|c|c|}
\hline \multirow{2}{*}{ Clinical procedures } & \multicolumn{2}{|c|}{ All patients } & \multicolumn{2}{|c|}{$\begin{array}{l}\text { Patients with either bilobar involvement } \\
\text { or AFP } \geq 400 \mathrm{ng} / \mathrm{mL}\end{array}$} \\
\hline & $\begin{array}{l}\text { Cost of PET/CT } \\
\text { for } 152 \text { patients }\end{array}$ & $\begin{array}{c}\text { Cost of } \\
\text { procedure avoided }\end{array}$ & $\begin{array}{l}\text { Cost of PET/CT } \\
\text { for } 74 \text { patients }\end{array}$ & $\begin{array}{l}\text { Cost of } \\
\text { procedure avoided }\end{array}$ \\
\hline PET/CT & $\$ 2,280,000$ & - & $\$ 1,065,000$ & - \\
\hline Hepatectomy and hospital stay & - & $\$ 712,320$ & - & $\$ 474,880$ \\
\hline Ablation and hospital stay & - & $\$ 82,760$ & - & $\$ 82,760$ \\
\hline Net cost & $\$ 2,280,000$ & $\$ 1,964,360$ & $\$ 1,110,000$ & $\$ 1,679,040$ \\
\hline
\end{tabular}

PET, positron emission tomography; CT, computed tomography; AFP, alpha-fetoprotein; TACE, transarterial chemoembolization.

of HCC. PET-CT made accurate diagnosis in all patients except one. Therefore, for patients with risk factors for HCC (e.g., hepatitis B or C virus, cirrhosis), newly diagnosed liver mass with atypical features of HCC should be treated as HCC and PET-CT should be for metastatic screening only.

In our patients with newly diagnosed HCC, $11.1 \%$ had metastatic disease. The rate should be much lower if another cohort with earlier disease had been included. The sites for HCC metastasis were lymph node, lung and bone. Less common sites, such as kidney and scalp, were not reported in our 17 patients who had metastasis.

Our findings echo previous studies in that high AFP and bilobar involvement are predictors for poor tumor biology, more aggressive tumor, high risk of metastasis, and hence poorer prognosis $(6,7)$. In our univariant analysis, pathological factors including poor liver function, large tumor and multifocal disease were also identified as predictive factors for PET-CT-detected metastasis. They were highly confounded with bilobar involvement and were thus adjusted in multivariant analysis.

At our center, we also manage patients with recurrent HCC. These lesions are usually smaller and with even more atypical features of HCC. They may mimic postoperative change, liver abscess, arteriovenous shunt or other liver tumor development. The value of PET-CT for this group of patients was not demonstrated in this study, and study on this topic will be valuable. Although the risk from radiation exposure to patients may be of less impact for the population known to have cancer because of their reduced life expectancy (8). Dual-tracer PET/CT was reported to have a radiation dose of $29 \mathrm{mSv}$ and up to about $57 \mathrm{mSv}$ if a 1-stop triple-phase contrast-enhanced CT was included. For patients included in the current study, PET/CT was utilized as a one-off initial workup. If repeated uses of dualtracer PET/CT were suggested as a surveillance imaging, the additional radiation burden needs to be considered and a judicious medical justification has to be made with every whole-body dual-tracer PET/CT referral (9).

In economical evaluation, the main goal is to compare two or more options by cost and result. However, rather than comparing PET/CT and an alternative traditional imaging modality, this study focused on the cost of management saved if PET/CT had been used to detect extrahepatic metastases, which is a similar study design as that by Heinrich et al. (10). In this sense, cost-benefit analysis would be most suitable since this approach does not require a "comparison" with an alternative imaging modality. Further details should include all related costs, e.g., the costs of extra-biochemical test, out-patient visits, etc. In real life, an inaccurate staging has a psychological adverse effect in addition to the financial cost. Economic analysis usually goes beyond pure effectiveness by merging the costs and consequences (altered management), and it is important that the "effectiveness" measure is generalizable and generally accepted (11). In this study, "diagnosis of metastasis" was considered a measure of effectiveness due to the high true positive value of $97 \%$.

In conclusion, dual-tracer PET/CT is useful for metastatic staging in HCC. Multivariable analysis showed that $\mathrm{AFP} \geq 400 \mathrm{ng} / \mathrm{mL}$ and bilobar involvement were independent predictive factors. With the application of the predictive factors, PET/CT was demonstrated to be more cost-effective in HCC workup.

\section{Acknowledgments}

Funding: None. 


\section{Footnote}

Reporting Checklist: The authors have completed the CHEERS reporting checklist. Available at https://hbsn. amegroups.com/article/view/10.21037/hbsn.2019.11.09/rc

Data Sharing Statement: Available at https://hbsn. amegroups.com/article/view/10.21037/hbsn.2019.11.09/dss

Conflicts of Interest: All authors have completed the ICMJE uniform disclosure form (available at https://hbsn. amegroups.com/article/view/10.21037/hbsn.2019.11.09/coif). Dr. CML and Dr. ACYC serve as the unpaid editorial board members of Hepatobiliary Surgery and Nutrition. The other authors have no conflicts of interest to declare.

Ethical Statement: The authors are accountable for all aspects of the work in ensuring that questions related to the accuracy or integrity of any part of the work are appropriately investigated and resolved. The study was conducted in accordance with the Declaration of Helsinki (as revised in 2013). This study has been approved by the Institutional Review Board of the University of Hong Kong/Hospital Authority Hong Kong West Cluster (IRB Reference Number: UW 19-315).

Open Access Statement: This is an Open Access article distributed in accordance with the Creative Commons Attribution-NonCommercial-NoDerivs 4.0 International License (CC BY-NC-ND 4.0), which permits the noncommercial replication and distribution of the article with the strict proviso that no changes or edits are made and the original work is properly cited (including links to both the formal publication through the relevant DOI and the license). See: https://creativecommons.org/licenses/by-nc-nd/4.0/.

\section{References}

1. Ferlay J, Shin HR, Bray F, et al. Estimates of worldwide

Cite this article as: Chu KKW, Chan ACY, Ma KW, She WH, Dai WC, Chok KSH, Cheung TT, Lo CM. Role of C11-FDG dual-tracer PET-CT scan in metastatic screening of hepatocellular carcinoma-a cost-effectiveness analysis. HepatoBiliary Surg Nutr 2021;10(3):301-307. doi: 10.21037/ hbsn.2019.11.09 burden of cancer in 2008: GLOBOCAN 2008. Int J Cancer 2010;127:2893-917.

2. Ho CL, Chen S, Yeung DW, et al. Dual-tracer PET/ CT imaging in evaluation of metastatic hepatocellular carcinoma. J Nucl Med 2007;48:902-9.

3. Ho CL, Yu SC, Yeung DW. 11C-acetate PET imaging in hepatocellular carcinoma and other liver masses. J Nucl Med 2003;44:213-21.

4. Hwang KH, Choi DJ, Lee SY, et al. Evaluation of patients with hepatocellular carcinomas using [(11)C]acetate and [(18)F]FDG PET/CT: a preliminary study. Appl Radiat Isot 2009;67:1195-8.

5. Cheung TT, Ho CL, Lo CM, et al. 11C-acetate and 18F-FDG PET/CT for clinical staging and selection of patients with hepatocellular carcinoma for liver transplantation on the basis of Milan criteria: surgeon's perspective. J Nucl Med 2013;54:192-200.

6. Chu KK, Chan SC, Fan ST, et al. Radiological prognosticators of hepatocellular carcinoma treated by hepatectomy. Hepatobiliary Pancreat Dis Int 2012;11:612-7.

7. Taketomi A, Toshima T, Kitagawa D, et al. Predictors of extrahepatic recurrence after curative hepatectomy for hepatocellular carcinoma. Ann Surg Oncol 2010;17:2740-6.

8. Brenner DJ, Shuryak I, Einstein AJ. Impact of reduced patient life expectancy on potential cancer risks from radiologic imaging. Radiology 2011;261:193-8.

9. Liu D, Khong PL, Gao Y, et al. Radiation dosimetry of whole-body dual-tracer 18F-FDG and 11C-acetate PET/CT for hepatocellular carcinoma. J Nucl Med 2016;57:907-12.

10. Heinrich S, Goerres GW, Schäfer M, et al. Positron emission tomography/computed tomography influences on the management of resectable pancreatic cancer and its cost-effectiveness. Ann Surg 2005;242:235-43.

11. Buck AK, Herrmann K, Stargardt T, et al. Economic evaluation of PET and PET/CT in oncology: evidence and methodologic approaches. J Nucl Med Technol 2010;38:6-17. 